\title{
Fettstoffwechselstörungen: Wo stehen wir - was wird kommen?
}

\begin{abstract}
Auch in diesem Jahr findet in Verbindung mit dem Tag des Cholesterins eine ärztliche Fortbildungsveranstaltung statt.
\end{abstract}

I- Die ärztliche Fortbildungsveranstal tung zum Thema "Fettstoffwechselstörungen: Wo stehen wir - was wird kommen?" der Deutschen Gesellschaft zur Bekämpfung von Fettstoffwechselstörungen und ihren Folgeerkrankungen DGFF (Lipid-Liga) e.V wird in Zusammenarbeit mit dem Diakoniekrankenhaus Mannheim sowie in Kooperation mit der Deutschen Gesellschaft für Arterioskleroseforschung (DGAF) e.V. durchgeführt.

Datum: Samstag, 18. Juni 2011, 9.30-14.00 Uhr
Ort: Diakoniekrankenhaus Mannheim, Festsaal im Mutterhaus, Speyerer Str. 91-93, 68163 Mannheim

Vorsitz: Dieter Schilling, Mannheim Achim Weizel, Mannheim

\footnotetext{
Themen:

- Fettstoffwechsel bei Diabetes mellitus Bedeutung und Behandlungsergebnisse (Karl Winkler, Freiburg)

- Sind Triglyzeride wirklich ein Risikofaktor für KHK? (Ulrich Laufs, Homburg)

- HDL - Was ist wichtiger: Funktion oder

Spiegel? (Jürgen Schäfer, Marburg)

- Pflanzensterole - Fluch oder Segen?

(Hans-Ulrich Klör, Gießen)

- Lipide: Karotisstenose und Stroke

(Klaus Amendt, Mannheim)
}

- Vitamin D-Mangel - ein neuer Risikofaktor für das Herz? (Winfried März, Eppelheim)

Für die Veranstaltung wurden bei der Akademie für ärztliche Fort- und Weiterbildung der Landesärztekammer BadenWürttemberg fünf CME-Punkte beantragt. Weitere Einzelheiten zum „Tag des Cholesterins" sind erhältlich über die Geschäftsstelle der DGFF und unter www.lipid-liga.de.

II DGFF (Lipid-Liga) e.V., Dr. rer. biol. hum. Heike Kantner, Präsidiumsmitglied DGAF-DGFF, Geschäftsführerin DGFF - koopt. Vorstandsmitglied Waldklausenweg 20, 81377 München Tel.: 089 / 7191001, Fax: 089 / 7142687 E-Mail: info@lipid-liga.de Internet: www.lipid-liga.de

\section{Zertifizierung von Tele-Stroke-Units} \section{Bessere Schlaganfallversorgung in ländlichen Gebieten}

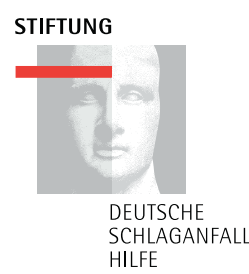

\begin{abstract}
In Deutschland verfügen etwa 180 Kliniken über eine zertifizierte Stroke Unit. Doch in ländlichen Regionen ist der Weg zur nächsten Stroke Unit häufig immer noch sehr weit. Eine telemedizinische Anbindung regionaler Krankenhäuser an überregionale Stroke Units kann hier Abhilfe schaffen.
\end{abstract}

Die telemedizinische Anbindung verbessert die Versorgung von Schlaganfallpatienten auf dem Land sowie deren Heilungsaussichten. Um die Qualität dieser telemedizinisch vernetzten Einrichtungen sicherzustellen, zertifizieren die Stiftung Deutsche Schlaganfall-Hilfe und die Deut- sche Schlaganfall-Gesellschaft (DSG) seit Januar 2011 auch sogenannte Tele-StrokeUnits. „Durch die Zertifizierung telemedizinischer Stroke Units ist die Umsetzung einer hochwertigen flächendeckenden Versorgung von Schlaganfallpatienten in greifbare Nähe gerückt", erklärt Dr. Brigitte Mohn, Vorsitzende des Vorstands der Stiftung Deutsche Schlaganfall-Hilfe. „Es ist unser Ziel, bei der Zertifizierung telemedizinischer Stroke Units an den Erfolg des bisherigen Qualitätssiegels anzuknüpfen."

„Die Zertifizierung von Tele-Stroke-Units ermöglicht es auch Krankenhäusern ohne neurologische Abteilung, eine Schlaganfallbehandlung auf hohem Niveau zu etablie- ren", sagte Prof. Otto Busse, Generalsekretär der DSG. Dennoch sei es nach wie vor das Ziel der DSG, in Deutschland eine weitgehend komplette Abdeckung mit neurologisch geleiteten Stroke Units zu erreichen.

Bisher konnten von der Deutschen Schlaganfall-Gesellschaft und der Stiftung Deutsche Schlaganfall-Hilfe etwa 180 Stroke Units in Deutschland zertifiziert werden. Der Einsatz moderner Informationstechnologien und das neue Zertifizierungsverfahren sollen die vorhandenen Versorgungslücken schließen.

\section{Weitere Informationen:} www.schlaganfall-hilfe.de 